In(Ga)As quantum dot formation on group-III assisted catalyst-free InGaAs nanowires

This article has been downloaded from IOPscience. Please scroll down to see the full text article.

2011 Nanotechnology 22195601

(http://iopscience.iop.org/0957-4484/22/19/195601)

View the table of contents for this issue, or go to the journal homepage for more

Download details:

IP Address: 128.178.106.14

The article was downloaded on 02/01/2012 at 15:52

Please note that terms and conditions apply. 


\title{
In(Ga)As quantum dot formation on group-III assisted catalyst-free InGaAs nanowires
}

\author{
Martin Heiss ${ }^{1,2}$, Bernt Ketterer $^{1}$, Emanuele Uccelli ${ }^{1,2}$, \\ Joan Ramon Morante ${ }^{3,4}$, Jordi Arbiol ${ }^{5}$ and \\ Anna Fontcuberta i Morral ${ }^{1,2}$ \\ ${ }^{1}$ Laboratoire des Matériaux Semiconducteurs, Institut des Matériaux, Ecole Polytechnique \\ Fédérale de Lausanne, Switzerland \\ ${ }^{2}$ Walter Schottky Institut and Physik Department, Technische Universität München, \\ Am Coulombwall 3, D-85748 Garching, Germany \\ ${ }^{3}$ Departament d'Electrònica, Universitat de Barcelona, E-08028 Barcelona, CAT, Spain \\ ${ }^{4}$ Catalonia Institute for Energy Research (IREC), E-08019 Barcelona, CAT, Spain \\ ${ }^{5}$ Institució Catalana de Recerca i Estudis Avançats (ICREA) and Institut de Ciència de \\ Materials de Barcelona, CSIC, 08193 Bellaterra, CAT, Spain \\ E-mail: anna.fontcuberta-morral@epfl.ch
}

Received 24 November 2010, in final form 27 January 2011

Published 23 March 2011

Online at stacks.iop.org/Nano/22/195601

\begin{abstract}
Growth of GaAs and $\operatorname{In}_{x} \mathrm{Ga}_{1-x}$ As nanowires by the group-III assisted molecular beam epitaxy growth method on $(001) \mathrm{GaAs} / \mathrm{SiO}_{2}$ substrates is studied in dependence on growth temperature, with the objective of maximizing the indium incorporation. Nanowire growth was achieved for growth temperatures as low as $550^{\circ} \mathrm{C}$. The incorporation of indium was studied by low temperature micro-photoluminescence spectroscopy, Raman spectroscopy and electron energy loss spectroscopy. The results show that the incorporation of indium achieved by lowering the growth temperature does not have the effect of increasing the indium concentration in the bulk of the nanowire, which is limited to $3-5 \%$. For growth temperatures below $575^{\circ} \mathrm{C}$, indium rich regions form at the surface of the nanowires as a consequence of the radial growth. This results in the formation of quantum dots, which exhibit spectrally narrow luminescence.
\end{abstract}

(Some figures in this article are in colour only in the electronic version)

\section{Introduction}

Nanowires are filamentary crystals with diameters of the order of few nanometers. Thanks to their peculiar shape and dimensions, they hold great promise for many technological advances in this century in diverse areas such as biosensing, energy harvesting and optoelectronics [1-5]. For the full deployment of such technology, control over the nanowire structure and composition at the nanometer scale is essential. In this respect, nanowire based heterostructures have been broadly studied $[2,3,6,7]$. Among the various compound semiconductors, InGaAs is considered to be one of the materials suitable as a transistor channel due to its low electron effective mass [8]. Additionally, InGaAs/GaAs based quantum wells and dots constitute the ideal material system for infrared detectors and single photon emitters. Moreover, the nanowire geometry has turned out to be ideal for all these applications as it enhances the functionality by allowing a better coupling with the electromagnetic radiation [9].

Nanowires are commonly obtained through the vaporliquid-solid (VLS; or vapor-solid-solid, VSS) method, where gold is used as a catalyst that preferentially gathers and decomposes the precursors $[10,11]$. As a result of the concerns raised by gold in the area of semiconductor technology, group III assisted growth has received increased attention [12-15]. This method is also compatible with the fabrication of heterostructures, for example by combining InGaAs/GaAs [16, 17] or zinc-blende/wurtzite crystal phases [18, 19]. 


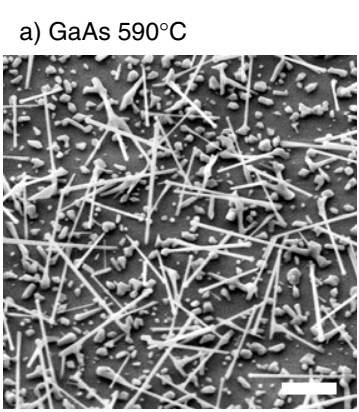

b) $\mathrm{GaAs} 575^{\circ} \mathrm{C}$

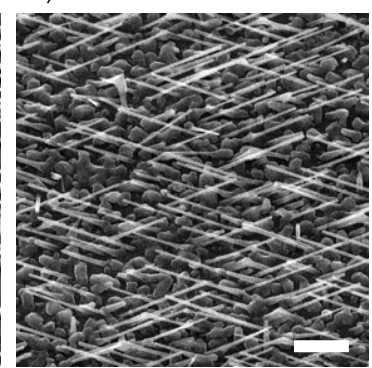

d) InGaAs $590^{\circ} \mathrm{C}$

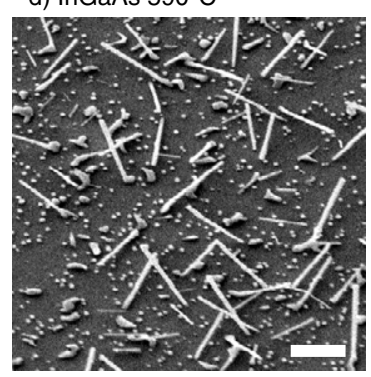

e) InGaAs $575^{\circ} \mathrm{C}$

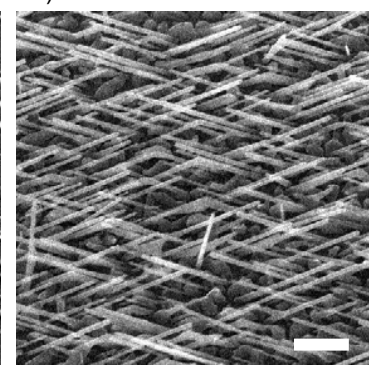

c) $\mathrm{GaAs} 560^{\circ} \mathrm{C}$

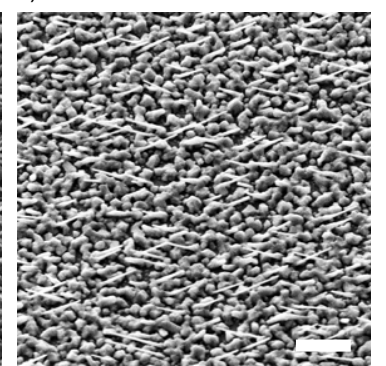

f) InGaAs $560^{\circ} \mathrm{C}$

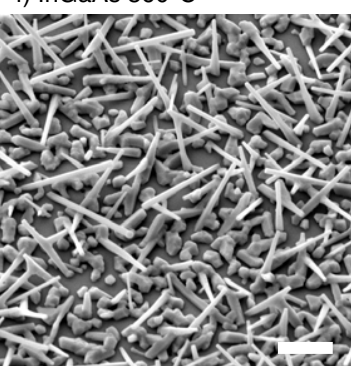

g) InGaAs $550^{\circ} \mathrm{C}$

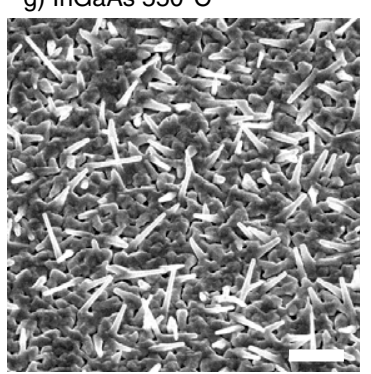

Figure 1. Representative tilted view (30 $)$ SEM micrographs of GaAs and $\operatorname{In}_{x} \mathrm{Ga}_{1-x}$ As nanowire samples grown at substrate temperatures of $550-590^{\circ} \mathrm{C}$ under otherwise identical conditions for the same growth times. The scale bar in each of the micrographs corresponds to $1 \mu \mathrm{m}$.

InGaAs and InAs quantum dots have been obtained in GaAs nanowires grown by the VLS method (Au assisted) [20]. Investigations on the optical properties and interface sharpness indicate that the InAs/GaAs system is more favorable than the InGaAs/GaAs [11, 21, 22]. The synthesis of InGaAs nanowires with the Au assisted method has been demonstrated by various groups. Martelli et al varied the growth temperature down to $480^{\circ} \mathrm{C}$, and reached an indium content up to $22 \%[23,24]$. However, the concentration turned out not to be homogeneous along the nanowire axis. Such inhomogeneities have been attributed to the longer diffusion length of indium with respect to GaAs and have also been observed by other groups $[6,25]$. InGaAs nanowires have also been obtained by selective area epitaxy. There, the difference in diffusion length between gallium and indium results in a different indium incorporation depending on the distance between the nanowires [26]. To date, it is still controversial as to what extent homogeneous InGaAs nanowires can be obtained and what is the maximum indium concentration [27]. The synthesis of InGaAs nanowire and InGaAs/GaAs nanowire heterostructures by the groupIII assisted method has been demonstrated recently [17]. In this case, the nanowire growth was performed under growth conditions optimized for the growth of GaAs nanowires. It was argued that due to the high growth temperatures, the incorporation of indium was limited to a few per cent. It is expected that a higher incorporation of indium may be reached by lowering the growth temperature. At the same time, previous studies have shown that the sticking coefficient of gallium on $\mathrm{SiO}_{2}$ increases as the substrate temperature is decreased and becomes close to unity for temperatures lower than $565^{\circ} \mathrm{C}$ [28]. Unfortunately, the optimum growth temperature range for the growth of $\operatorname{In}_{x} \mathrm{Ga}_{1-x}$ As compounds corresponds to temperatures below $520^{\circ} \mathrm{C}$ [29]. Indeed, by lowering the growth temperature down to the range of $400-505^{\circ} \mathrm{C}$, pure InAs growth was obtained [30, 31]. The challenge in catalyst-free growth is then to find conditions where gallium still participates in the catalyst-free growth and where the incorporation of indium is enhanced. The first condition would normally require a growth temperature between 600 and $630^{\circ} \mathrm{C}$. However, a larger incorporation of indium is only expected at considerably lower growth temperatures. In this work we study the influence of the growth temperature on the growth of InGaAs nanowires in the range between the optimum growth temperature windows for pure GaAs and pure InAs nanowire growth. We discuss the implications in terms of indium incorporation and optical properties.

\section{Experimental details}

The nanowires were grown by the group-III assisted method by molecular beam epitaxy as detailed elsewhere [14, 17, 32]. For this, (001) oriented GaAs substrates covered with a thin layer of $\mathrm{SiO}_{2}$ were used. A systematic growth temperature series, where only gallium or both gallium and indium were supplied during the entire growth process, was realized. The growth temperature series for pure GaAs nanowires grown under the same conditions is used as a reference. The substrate temperature was varied in the range of $550-590{ }^{\circ} \mathrm{C}$. The indium and gallium growth rates were fixed at $0.045 \AA \mathrm{s}^{-1}$ and $0.2 \AA \mathrm{s}^{-1}$, respectively. The $\mathrm{As}_{4}$ beam flux was set to $8.8 \times 10^{-7}$ mbar at a constant growth time of $5400 \mathrm{~s}$ for all samples. Further details on the growth procedure can be found in [32]. The morphology of the nanowires was studied by scanning electron microscopy (SEM). The composition and crystal structure of the nanowires was studied by high resolution electron microscopy (HRTEM), electron energy loss spectroscopy (EELS) and Raman spectroscopy using the 
$488 \mathrm{~nm}$ line of an $\mathrm{Ar}^{+} \mathrm{Kr}^{+}$-laser focused to a diffraction limited spot (numerical aperture $=0.75$, power density $50 \mathrm{~kW} \mathrm{~cm}^{-2}$ ). The optical properties of single nanowires were studied by photoluminescence spectroscopy at $4.2 \mathrm{~K}$ with a confocal microscope or at $10 \mathrm{~K}$ in a He flow cryostat. The photoluminescence was excited with a $780 \mathrm{~nm}$ diode-laser or a $633 \mathrm{~nm}$ HeNe-laser focused to a diffraction limited spot (numerical aperture $=0.65$ ). For this, the nanowires were transferred from the original substrate to a silicon substrate with a $50 \mathrm{~nm} \mathrm{SiO}_{2}$ layer.

\section{Effect of the growth temperature on the morphology of GaAs and $\operatorname{In}_{x} \mathrm{Ga}_{1-x}$ As nanowires}

We start by discussing the morphology of the nanowires grown under the different conditions. Representative SEM micrographs are shown in figure 1. GaAs and InGaAs nanowires could be obtained down to a temperature of $550{ }^{\circ} \mathrm{C}$. By lowering the growth temperature, we observe an increase in the material deposited between the nanowires. These are randomly oriented GaAs deposits that nucleate on the oxide due to the decrease in the mobility of $\mathrm{Ga}$ on the surface when the substrate temperature is reduced. Besides the GaAs sample grown at $560^{\circ} \mathrm{C}$, neither the amount of surface deposits or the length of the nanowires show significant deviations compared to the pure GaAs samples grown under otherwise identical conditions (figures 1(a)-(c)). This indicates that the sticking coefficient is not significantly altered by the presence of In:Ga with a beam flux ratio of 1:4 as compared to the situation with a pure gallium supply. At $560{ }^{\circ} \mathrm{C}$, almost the entire surface between the nanowires is covered with randomly oriented (In)GaAs deposits (see figures 1(c), (f) and $(\mathrm{g}))$. The typical size of these structures is up to $500 \mathrm{~nm}$. Concurrently, the length of the nanowires is reduced with regard to figures 1(a)-(c). This is also consistent with a reduced surface diffusion of gallium that is incorporating in the surface deposits in the case of a low growth temperature. Generally, the observed temperature dependence is in agreement with previous studies for the sticking coefficient of gallium on such $\mathrm{SiO}_{2}$ surfaces [28], which demonstrated a sticking coefficient of unity for temperatures below $565^{\circ} \mathrm{C}$.

One should note that some of the samples did not show an alignment of the nanowires toward the $[1 \overline{1} 1] B$ or $[11 \overline{1}] B$ directions of the GaAs(100) growth substrate.

\section{Optical properties of the $\operatorname{In}_{x} \mathrm{Ga}_{1-x}$ As nanowires}

We expect that the lowering of the growth temperature should have a direct influence on the indium content and therefore on the optical properties. Upon homogeneous incorporation of indium, the band gap $E_{\mathrm{g}}$ should decrease in the form [33]:

$$
E_{\mathrm{g}}\left(\operatorname{In}_{x} \mathrm{Ga}_{1-x} \mathrm{As}\right)=1.5192-1.5837 x+0.475 x^{2}(\mathrm{eV}) .
$$

Figure 2(a) summarizes the results of the microphotoluminescence experiments performed at $4.2 \mathrm{~K}$ on the $\mathrm{In}_{x} \mathrm{Ga}_{1-x}$ As nanowires. For completion, we have added the data point from a previous study of an $\mathrm{InGaAs}$ nanowire grown
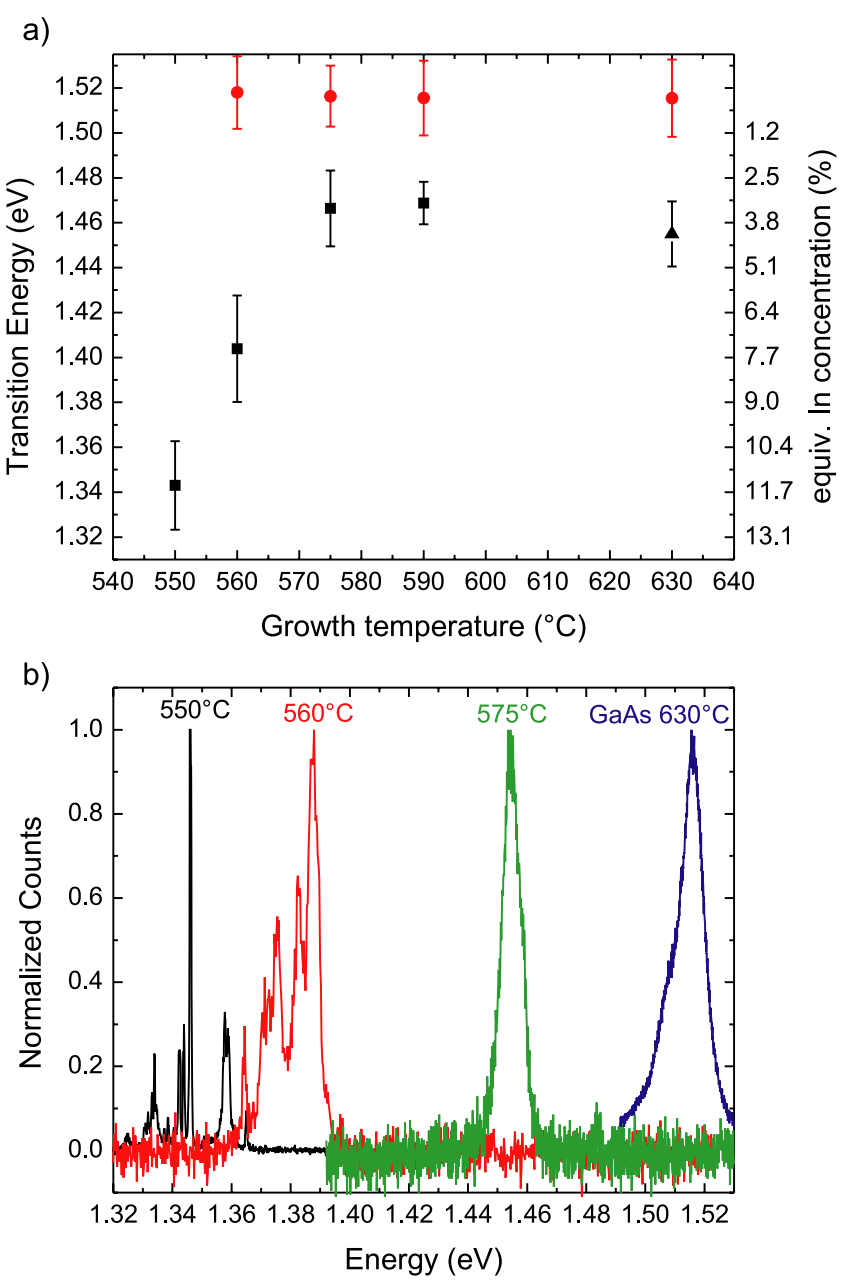

Figure 2. (a) Typical $4.2 \mathrm{~K}$ micro-photoluminescence transitions for $\mathrm{In}_{x} \mathrm{Ga}_{1-x}$ As nanowire samples grown at temperatures of $550-590^{\circ} \mathrm{C}$. - correspond to data from this work while $\boldsymbol{\Lambda}$ corresponds to data from a previous study [17] using a higher In deposition rate of $0.088 \AA \mathrm{s}^{-1}$. The red dots $(\bullet)$ show for reference the $10 \mathrm{~K}$ micro-photoluminescence transitions observed in single pure GaAs nanowires grown at temperatures of $560-630^{\circ} \mathrm{C}$. The right axis of the graph shows the indium concentration of an idealized $\mathrm{In}_{x} \mathrm{Ga}_{1-x}$ As material with a band gap related to the transition energy. (b) Typical $4.2 \mathrm{~K}$ photoluminescence spectra for $\operatorname{In}_{x} \mathrm{Ga}_{1-x} \mathrm{As}$ nanowire samples grown at temperatures of 550,560 and $575^{\circ} \mathrm{C}$ under otherwise identical conditions. For reference the spectrum of a pure GaAs nanowire synthesized at $630^{\circ} \mathrm{C}$ under otherwise identical conditions is shown.

at $630{ }^{\circ} \mathrm{C}$ using a higher In deposition rate of $0.088 \AA \mathrm{s}^{-1}$ [17]. Otherwise this sample was grown under identical conditions for the same growth time [17]. Figure 2(a) shows the average photoluminescence peak position obtained from various single nanowires obtained on the same growth run. The error bars indicate the standard deviation of peak positions of the measurements on various nanowires. For comparison figure 2(a) shows the $10 \mathrm{~K}$ photoluminescence transitions for the pure GaAs nanowire samples synthesized under otherwise identical conditions. The lower limit for the indium concentration $x$ in the $\operatorname{In}_{x} \mathrm{Ga}_{1-x}$ As nanowires estimated by equation (1) is indicated on the right axis. One should note that 
a)

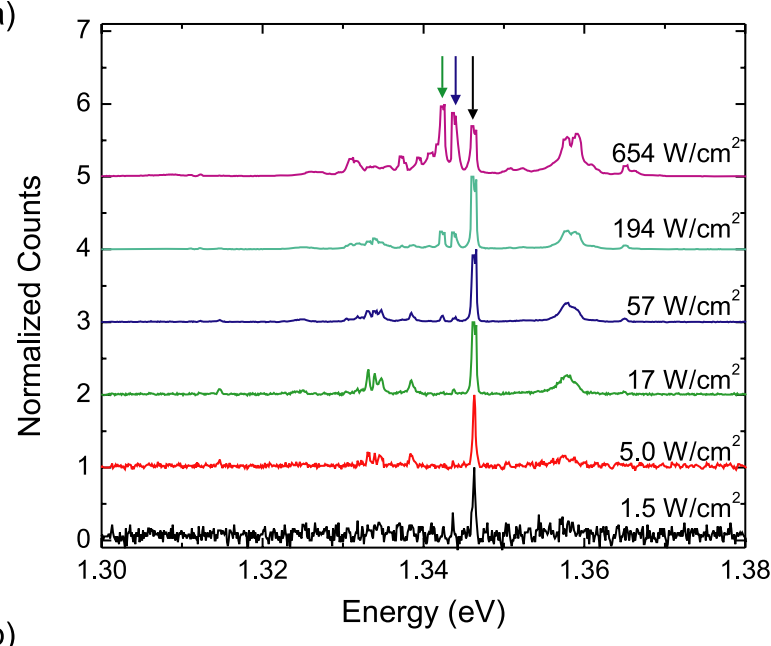

b)

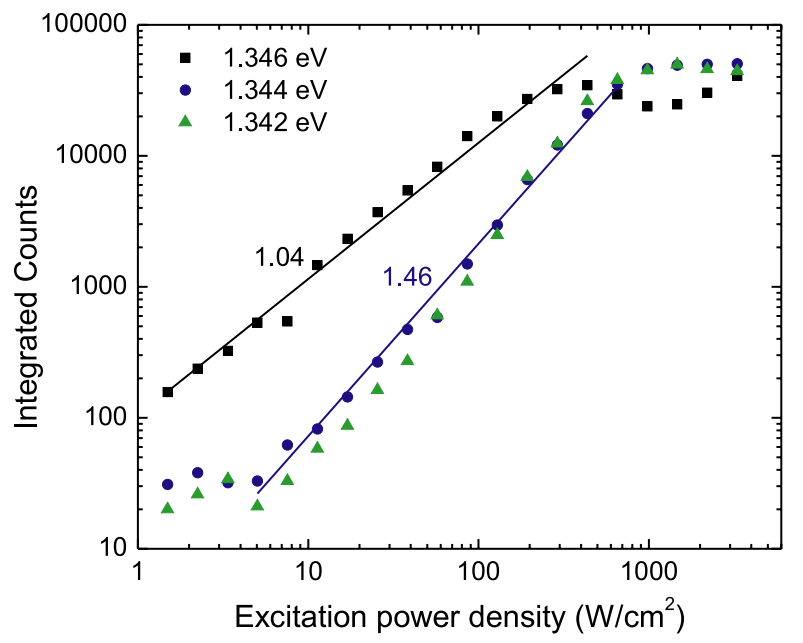

Figure 3. (a) Normalized $4.2 \mathrm{~K}$ photoluminescence spectra of a $\mathrm{In}_{x} \mathrm{Ga}_{1-x} \mathrm{As}$ nanowire sample grown at $550{ }^{\circ} \mathrm{C}$ for several different excitation power densities. (b) Excitation power dependence of the three spectrally narrow emission lines marked by the colored arrows in (a).

equation (1) does not consider quantum confinement effects and could therefore underestimate the actual indium content of the optically emitting structures. We observe a red shift in the photoluminescence transitions with respect to pure GaAs, which is a clear indication of the presence of indium in the nanowires. Surprisingly, the peak position does not change significantly for nanowires obtained at temperatures from $630^{\circ} \mathrm{C}$ down to $575^{\circ} \mathrm{C}$. In all these cases, the optical properties of the nanowires correspond to those of an indium concentration of only $\approx 3 \%$. As a consequence, we can state that the indium incorporation in the nanowire is not affected by the growth temperature within a variation in this range of temperature. This observation is in quantitative disagreement with thermodynamic calculations of Shen and Chatillon [29]. However, these thermodynamic calculations [29] also show that $\mathrm{In}_{0.01} \mathrm{Ga}_{0.99} \mathrm{As}$ is thermodynamically more stable under vacuum than pure GaAs. This qualitatively explains the remaining small indium incorporation for the high growth temperature regime. The quantitative deviation could indicate



Figure 4. Raman spectra of single $\operatorname{In}_{x} \mathrm{Ga}_{1-x}$ As nanowires from the samples grown at temperatures of $550-590{ }^{\circ} \mathrm{C}$. The peak marked with $(*)$ corresponds to a line of the laser.

that the incorporation of indium might not be exclusively governed by the VLS mechanism through the droplet. For growth temperatures below $575^{\circ} \mathrm{C}$ a pronounced redshift of the emission is observed. In principle, this gives an indication that the incorporation of indium in the nanowire may be significantly increased by reducing the growth temperature. However, in order to have more precise information on the incorporation of indium, we perform a more detailed analysis.

Typical spectra of the InGaAs nanowires obtained under different temperatures are shown in figure 2(b). Interestingly, the decrease in the growth temperature is accompanied with an increase in the variations in photoluminescence characteristics from nanowire to nanowire. At the same time, we also observe that the shape of the photoluminescence spectra changes for growth temperatures below $575^{\circ} \mathrm{C}$. This is illustrated in figure 2(b). Nanowires grown between 630 and $575^{\circ} \mathrm{C}$ typically exhibit a quite narrow single emission peak around $1.46 \mathrm{eV}$. The nanowires grown at lower temperatures present spectra with multiple peaks. Furthermore, the photoluminescence emission of these nanowires is typically inhomogeneous along the nanowire axis. The most extreme example of this was observed in a sample grown at $550^{\circ} \mathrm{C}$. A photoluminescence spectrum of this sample is shown in figure 3(a). In this case, the emission under low excitation powers consists mainly of a single spectrally narrow line. At higher excitation powers two additional narrow lines and several other peaks appear. The main emission line at $1.346 \mathrm{eV}$ has a full width at half maximum of only $365 \mu \mathrm{eV}$. This spectral width is at the limit of the resolution of our spectrometer. In order to better understand the nature of these 

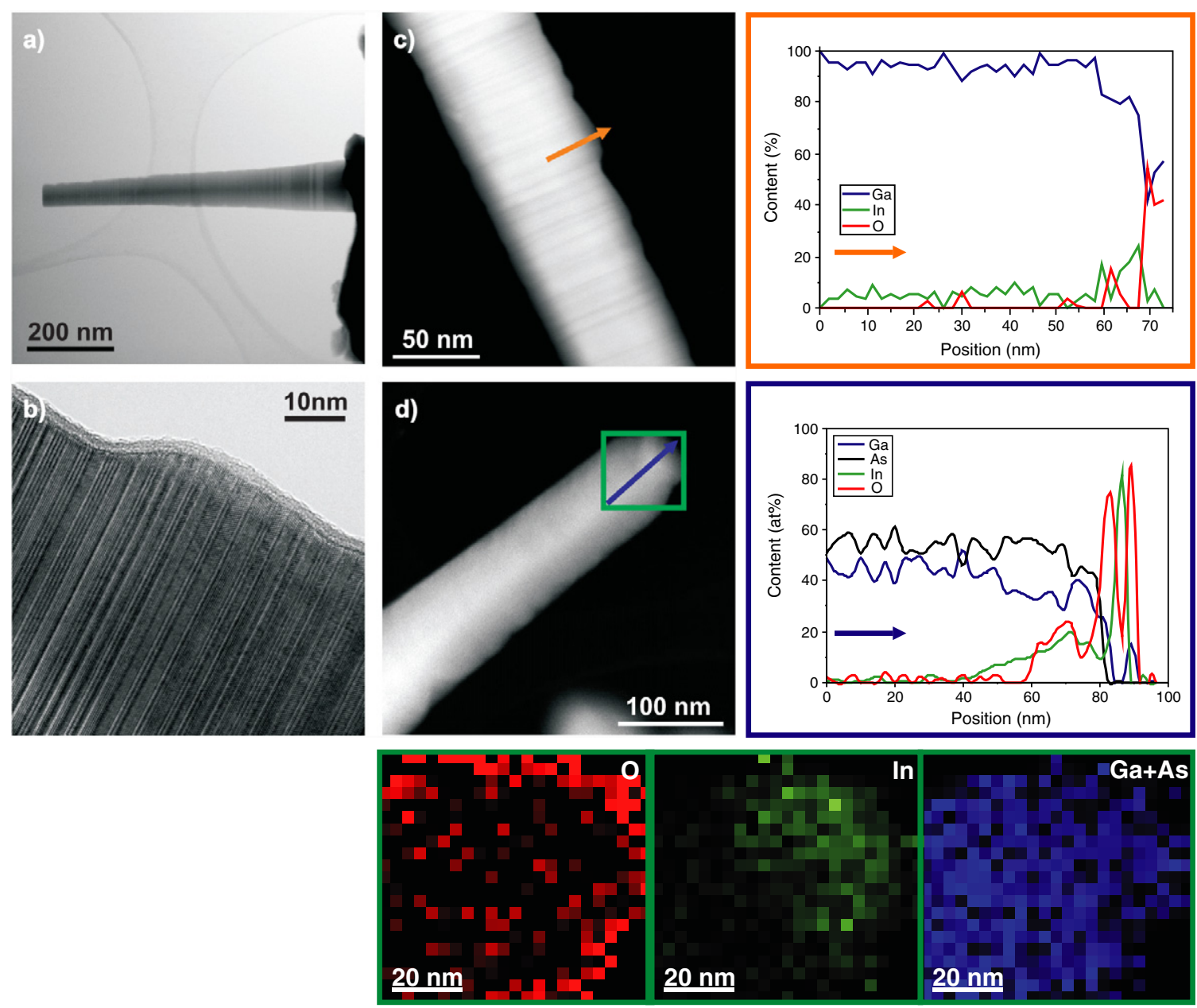

Figure 5. (a) Bright field STEM micrograph of a nanowire from the $\operatorname{In}_{x} \mathrm{Ga}_{1-x}$ As sample grown at $550^{\circ} \mathrm{C}$. (b) High resolution micrograph of a nanowire, showing accumulations of material on the surface. (c) EELS profile perpendicular to the nanowire growth direction clearly showing a higher concentration of indium on the NW side facets. (d) EELS profile along the growth direction at the tip of a nanowire. EELS spectrum imaging mapping of the tip region, showing the indium rich tip of this nanowire. For the analysis the indium $\mathrm{M}_{4,5}(443 \mathrm{eV})$, the oxygen $\mathrm{K}$ edge $(532 \mathrm{eV})$, the gallium $\mathrm{L}_{2,3}$ edge and the arsenic $\mathrm{L}_{2,3}$ edge are used.

transitions, we measured the photoluminescence spectra as a function of the excitation power. The integrated intensity of the lines as a function of the excitation power is shown in figure 3(b). The main emission line at $1.346 \mathrm{eV}$ has a linear excitation power dependence, while the emission peaks at 1.344 and $1.342 \mathrm{eV}$ have a nonlinear power dependence $\propto P^{1.46}$ as illustrated by the slope of the linear fit in the double logarithmic plot of figure 3(b). Such behavior is typically only observed in low-dimensional systems such as quantum dots [34]. Interestingly, in order to achieve such a quantum dot like zero-dimensional confinement, the related structures must actually be of dimensions much smaller than the diameter of the nanowires. The existence of quantum dots in the nanowire structure may be related to inhomogeneities in the indium concentration at lower temperatures. In order to understand if this is the case, a more detailed analysis of the structure and composition is essential. Therefore, we realized a detailed study on the structure and indium distribution in the nanowires. The results are shown in section 5 .

\section{Structural characteristics of $\operatorname{In}_{x} \mathrm{Ga}_{1-x} \mathrm{As}$ nanowires}

The frequency positions of the optical phonons in the ternary compound $\mathrm{In}_{x} \mathrm{Ga}_{1-x}$ As strongly depend on the compositional fraction $x$ [35]. Therefore, measuring the Raman shift of the optical phonons provides a convenient non-destructive method for estimating the composition. In figure 4 Raman spectra of the $\operatorname{In}_{x} \mathrm{Ga}_{1-x}$ As nanowires grown at various temperatures are shown. For the sample grown at $590^{\circ} \mathrm{C}$, the width and the position of the transverse optical mode at $267.5 \mathrm{~cm}^{-1}$ shows no observable deviations from the pure binary GaAs compound within the experimental uncertainty. By decreasing the growth temperature, we observe a continuous broadening and down shift of the GaAs-like optical phonons. This is directly related to an increase in the indium content in the nanowire. Interestingly, for the nanowire grown at $560^{\circ} \mathrm{C}$ we observe two overlapping features in the spectra: a broad background denoted by an arrow in figure 4 and a superimposed sharper TO mode. This indicates a non-homogeneous distribution 
a)

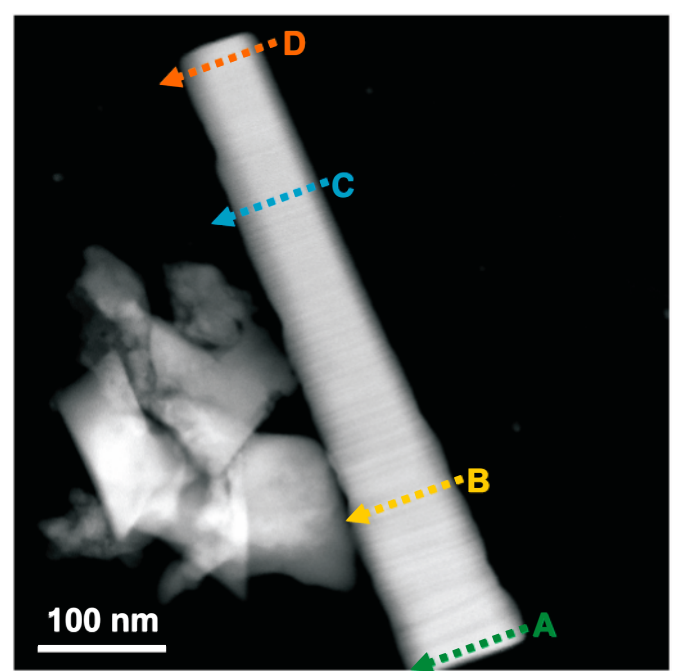

b)

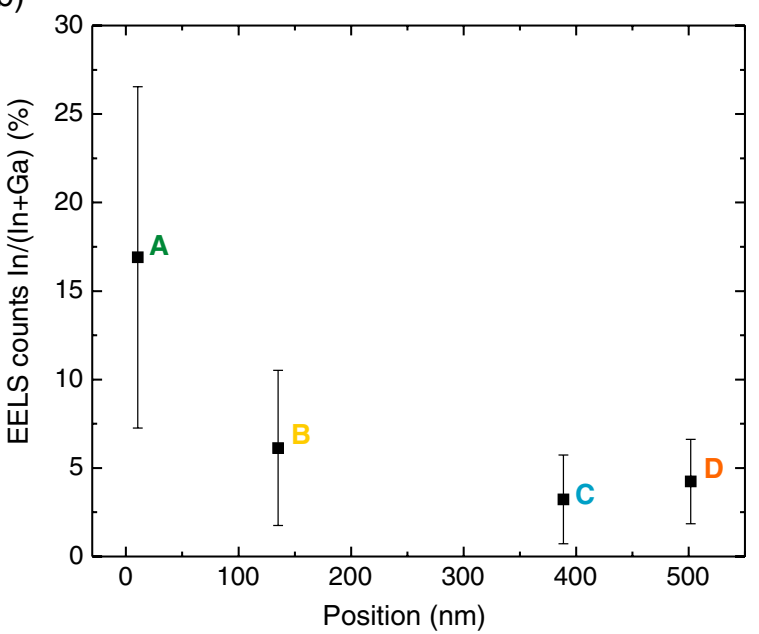

Figure 6. (a) ADF-STEM micrograph of a nanowire grown at $550^{\circ} \mathrm{C}$. (b) Indium content obtained from the averaged EELS line scan profiles A-D marked in (a).

of In in the nanowire. We suggest that this is due to the formation of a shell with higher In content around the core GaAs nanowire. For the employed laser wavelength of $488 \mathrm{~nm}$ the Raman information depth is in the order of $40 \mathrm{~nm}$. For the sample grown at $550^{\circ} \mathrm{C}$, a down shift of the TO mode of $6.9 \mathrm{~cm}^{-1}$ is observed. From this shift, in comparison with literature data [36], we can estimate an indium concentration up to $23 \%$ in these nanowires. The spatial resolution of the Raman spectroscopy is not sufficient to obtain information on the axial distribution of indium in these nanowires that are only approximately $700 \mathrm{~nm}$ long.

In order to provide a more detailed analysis of spatial inhomogeneities of indium incorporation and at the same time the exact origin of the quantum dot like photoluminescence features observed for the nanowires, the crystalline structure and indium composition of the sample grown at $550^{\circ} \mathrm{C}$ were analyzed with transmission electron microscopy and EELS. The results are presented in figure 5. Figure 5(a) shows the bright field STEM micrograph of a typical nanowire of the sample. The nanowires have a length in the order of $700 \mathrm{~nm}$ and exhibit tapering. In figure 5(b) a high resolution STEM micrograph is shown. The nanowires consist of a highly twinned zinc-blende structure with the sporadic inclusion of some wurtzite segments with thicknesses up to $10 \mathrm{~nm}$. The surface of the nanowire is oxidized, the oxide thickness being of the order of $2 \mathrm{~nm}$. Moreover, the side facets of the nanowire are not perfectly flat as we have observed in the past for pure GaAs nanowires $[14,16]$. They present some rounded structures (dome-like), which have a typical height and length, respectively, of $5 \mathrm{~nm}$ and $50 \mathrm{~nm}$. In order to obtain more information on the nature of these domes, we did EELS scans along the diameter of the nanowire at several points. The result is shown in figure 5(c). The core of the nanowire is composed of InGaAs with an indium concentration of 3-5\%. Interestingly, the indium concentration increases up to approximately $20 \%$ at the surface, coinciding with the formation of the nano-domes. We believe that the nano-domes formed at the surface of the nanowire are most likely the origin of the spectrally narrow emission lines in the photoluminescence characteristics. We would like to point out that the dimensions of the nano-domes are similar to those observed in Stranski-Krastanov quantum dots [37].

Now we would like to discuss the growth mechanisms of the indium rich nano-domes formed on the surface of the nanowires. Figure 6(a) clearly shows that the nanowires grown at $550^{\circ} \mathrm{C}$ are tapered. This is a clear indication of radial growth. If the formation of indium rich regions is related to the radial growth, one should find a gradient in the indium concentration at the surface. For this purpose, we have realized EELS scans along the nanowire diameter at different points shown in figure 6 . We have found that indeed the formation of indium rich regions is inhomogeneous along the nanowire and the concentration is higher at the bottom of the nanowire. Formation of InGaAs quantum dots on GaAs(110) surfaces has been observed in the past for similar growth temperatures [38]. Indeed this could account for the spectrally narrow features observed in the sample grown at $550^{\circ} \mathrm{C}$.

Finally, we have realized an EELS spectroscopy map of the region close to the tip of a nanowire. This is illustrated in figure 5(d). At the nanowire droplet at the tip, a peak indium concentration as high as $80 \%$ is observed. This clearly shows that the catalyst droplet is very rich in indium during the growth. In the final $40 \mathrm{~nm}$ below the tip, the indium concentration in the solid $\operatorname{In}_{x} \mathrm{Ga}_{1-x}$ As gradually increases to a concentration of up to $x \approx 40 \%$. We believe that the increased incorporation is related to the final part of the growth, when substrate heating is stopped. At that stage, there is still some residual axial growth as the arsenic pressure is maintained. This indicates that a higher indium incorporation during the axial growth might be obtained by lowering the growth temperature even further.

\section{Conclusions}

The formation of $\operatorname{In}_{x} \mathrm{Ga}_{1-x}$ As heterostructures in nanowires by catalyst-free molecular beam epitaxy has been studied in dependence on growth temperature. The incorporation of indium in the nanowire core was shown to be limited to 
3-5\%. A growth temperature series showed that for temperatures below $575^{\circ} \mathrm{C}$ indium incorporation occurs predominantly through radial growth, as demonstrated by a detailed EELS analysis. The optical properties of such structures resulted in spectrally narrow peaks and an excitation power dependence typical of quantum dots.

\section{Acknowledgments}

The authors acknowledge the experimental support of G Abstreiter and M Bichler. Funding through the Marie Curie Excellence Grant SENFED, SFB631, Nanosystems Initiative Munich, ERC Starting Grant Upcon and SNF 129775 is also greatly acknowledged. This work was partially supported by the Spanish Government projects Consolider Ingenio 2010 CSD2009 00013 IMAGINE and CSD2009 00050 MULTICAT. JA acknowledges funding from the MICINN project MAT2010-15138 (COPEON) and NanoAraCat. The authors would like to thank the TEM facilities in Instituto de Nanociencia de Aragón (INA).

\section{References}

[1] Cui Y, Duan X, Hu J and Lieber C M 2000 J. Phys. Chem. B $1045213-6$

[2] Cui Y and Lieber C M 2001 Science 291 851-3

[3] Samuelson L, Björk M T, Deppert K, Larsson M, Ohlsson B J, Panev N, Persson A I, Sköld N, Thelander C and Wallenberg L R 2004 Physica E 21 560-7

[4] Wang W U, Chen C, Lin K H, Fang Y and Lieber C M 2005 Proc. Natl Acad. Sci. USA 102 3208-12

[5] Kayes B M, Atwater H A and Lewis N S 2005 J. Appl. Phys. 97114302

[6] Cornet D M and LaPierre R R 2007 Nanotechnology 18385305

[7] Dick K A, Kodambaka S, Reuter M C, Deppert K, Samuelson L, Seifert W, Wallenberg L R and Ross F M 2007 Nano Lett. 7 1817-22

[8] Vurgaftman I, Meyer J R and Ram-Mohan L R 2001 J. Appl. Phys. 89 5815-75

[9] Borgström M T, Zwiller V, Müller E and Imamoglu A 2005 Nano Lett. 5 1439-43

[10] Dick K A, Deppert K, Martensson T, Mandl B, Samuelson L and Seifert W 2005 Nano Lett. 5 761-4

[11] Paladugu M, Zou J, Guo Y N, Zhang X, Kim Y, Joyce H J, Gao Q, Tan H H and Jagadish C 2008 Appl. Phys. Lett. 93101911

[12] Yazawa M, Koguchi M and Hiruma K 1991 Appl. Phys. Lett. 58 1080-2

[13] Novotny C J and Yu P K L 2005 Appl. Phys. Lett. 87203111
[14] Fontcuberta i Morral A, Colombo C, Abstreiter G, Arbiol J and Morante J R 2008 Appl. Phys. Lett. 92063112

[15] Plissard S, Dick K A, Larrieu G, Godey S, Addad A, Wallart X and Caroff P 2010 Nanotechnology 21385602

[16] Uccelli E, Arbiol J, Morante J R and Fontcuberta i Morral A 2010 ACS Nano 4 5985-93

[17] Heiß M, Gustafsson A, Conesa-Boj S, Peiro F, Morante J R, Abstreiter G, Arbiol J, Samuelson L and Fontcuberta i Morral A 2009 Nanotechnology 20075603

[18] Zardo I, Conesa-Boj S, Peiro F, Morante J R, Arbiol J, Uccelli E, Abstreiter G and Fontcuberta i Morral A 2009 Phys. Rev. B 80245324

[19] Spirkoska D et al 2009 Phys. Rev. B 80245325

[20] Gunawan A A, Jha S and Kuech T F 2010 J. Vac. Sci. Technol. B 28 1111-9

[21] Panev N, Persson A I, Skold N and Samuelson L 2003 Appl. Phys. Lett. 83 2238-40

[22] Krogstrup P, Yamasaki J, Sørensen C B, Johnson E, Wagner J B, Pennington R, Aagesen M, Tanaka N and Nygård J 2009 Nano Lett. 9 3689-93

[23] Jabeen F, Rubini S, Grillo V, Felisari L and Martelli F 2008 Appl. Phys. Lett. 93083117

[24] Jabeen F, Rubini S and Martelli F 2009 WRA-LDSD Workshop of Recent Advances on Low Dimensional Structures and Devices; Microelectron. J. $40442-5$

[25] Jasik A, Wnuk A, Gaca J, Wójcik M, Wójcik-Jedlinska A, Muszalski J and Strupinski W 2009 J. Cryst. Growth 311 4423-32

[26] Sato T, Motohisa J, Noborisaka J, Hara S and Fukui T 2008 J. Cryst. Growth 310 2359-64

[27] Sudfeld D, Regolin I, Kästner J, Dumpich G, Khorenko V, Prost W and Tegude F J 2006 Phase Translt. A 79 727-37

[28] Heiß M, Riedlberger E, Spirkoska D, Bichler M, Abstreiter G and Fontcuberta i Morral A 2008 J. Cryst. Growth 310 1049-56

[29] Shen J Y and Chatillon C 1990 J. Cryst. Growth 106 543-52

[30] Koblmüller G, Hertenberger S, Vizbaras K, Bichler M, Bao F, Zhang J P and Abstreiter G 2010 Nanotechnology 21365602

[31] Colombo C 2007 Nucleation and growth mechanisms of catalyst-free GaAs nanowires by molecular beam epitaxy Master Thesis Walter Schottky Institute

[32] Colombo C, Spirkoska D, Frimmer M, Abstreiter G and Fontcuberta i Morral A 2008 Phys. Rev. B 77155326

[33] Goetz K H, Bimberg D, Jurgensen H, Selders J, Solomonov A V, Glinskii G F and Razeghi M $1983 \mathrm{~J}$. Appl. Phys. 54 4543-52

[34] Brunner K, Abstreiter G, Böhm G, Tränkle G and Weimann G 1994 Phys. Rev. Lett. 73 1138-41

[35] Brodsky M H and Lucovsky G 1968 Phys. Rev. Lett. 21 990-3

[36] Islam M R, Verma P, Yamada M, Tatsumi M and Kinoshita K 2002 Japan. J. Appl. Phys. 41 991-5

[37] Wasilewski Z R, Fafard S and McCaffrey J P 1999 J. Cryst. Growth 201/202 1131-5

[38] Blumin M, Ruda H E, Savelyev I G, Shik A and Wang H 2006 J. Appl. Phys. 99093518 